\title{
PHASE TRANSITION BEHAVIOUR OF ISING SPIN GLASS ON THE FCC LATTICE
}

\author{
NGO VAN THANH \\ Institute of Physics, Vietnam Academy of Science and Technology, \\ 10 Dao Tan, Ba Dinh, Hanoi, Vietnam \\ HOANG DINH TIEN \\ High School for Gifted Students, Vinh University, \\ 182 Le Duan, Vinh city, Nghe An province, Vietnam \\ DIEP THE HUNG \\ Cergy-Pontoise University, CNRS UMR 8089 \\ 2, Avenue Adolphe Chauvin, 95302 Cergy-Pontoise Cedex, France \\ Email: nvthanh@iop.vast.ac.vn \\ Received 09 July 2013; \\ Accepted for publication 31 August 2013
}

\begin{abstract}
In this work, we study the nature of phase transition in a face centered cubic (FCC) antiferromagnet with Ising spins. The spin-glass character depends on the concentration $p$ of ferromagnetic bonds randomly generated into the system. We introduce a new quantity $M_{Q}$ combined by the Edwards-Anderson order parameter $Q$ and the standard magnetization $M$. Note that, it is impossible to obtain the susceptibility defined by the variance of $Q$ or $M$, but we can do that for $M_{Q}$. Using the standard Monte-Carlo and powerful Wang-Landau flat-histogram methods, we carry out in this work intensive simulations with many value of $p$. We show that the first-order transition has been destroyed with a tiny amount of ferromagnetic bond $p \sim 0.01$. With increasing $p$, the antiferromagnetic phase changes into a spin glass, and then to ferromagnetic phase.
\end{abstract}

\section{INTRODUCTION}

A spin glass is a disordered magnet with random interactions, both ferromagnetic and also antiferromagnetic bonds are randomly distributed with comparable frequency. The term "glass" comes from an analogy between the magnetic disorder in a spin glass and the positional disorder of a conventional, chemical glass, a window glass. Spin glasses have many metastable structures, so it is very difficult to explore experimentally or in simulations.

Phase transition in spin glasses is a subject of intensive investigation since four decades. The main difficulties in such systems come from the combination of the frustration and the bond disorder. The most popular Ising spin glass model have been introduced by Sherrington and Kirkpatrick [1,2]. This is a classical mean field model with random interactions between the spin pairs. 
The phenomena of slow dynamics or slow relaxation near the phase transition have been the subject of many theoretical [3-6] and experimental [7-10] studies, it presents many interesting features that reflect the nature of the phase transition.

The paper is organized as follows. Sec. II is devoted to a description of our model and the simulation method. Results are shown in Sec. III. Conclusions are given in Sec. IV.

\section{THE MODEL}

We consider the FCC lattice with Ising spins of magnitude $S=1$. The Hamiltonian is given by

$$
H=-\sum_{<i, j>} J_{i j}\left(S_{i} S_{j}\right)
$$

where $S_{i}$ is the Ising spin at the lattice site $i, \sum_{(i, j)}$ is made over the NN spin pairs $S_{i}$ and $S_{j}$ with interaction $J_{i j}$. Hereafter we suppose that $J_{i j}=-J(J>0)$ for antiferromagnetic bonds and $J_{i j}=J$ for ferromagnetic bonds. The three-dimension (3D) lattice is composed of $L^{3}$ FCC-cells each with four spins ( $L$ being the number of cells in each direction). The four sublattices are shown in Fig. 1. The total number of spins of the lattice is $N=4 L^{3}$. The number of NN bond per spin is 12 , thus the total number of bond of the system is $N_{b}=12 \times N / 2=24 L^{3}$. We use the periodic boundary conditions.

To create a spin glass, we introduce a number of ferromagnetic bonds $N_{b}^{F}$ in a random manner into the pure antiferromagnetic state. We define the ferromagnetic bond concentration $p$ by $p=N_{b}^{F} / N_{b}$. The phase transition behavior of the system depends on the parameter $p(0 \leq p \leq 1)$, i.e., it changes from pure antiferromagnetic phase (at $p=0)$ to pure ferromagnetic phase at $(p=1)$ passing through the spin glass phase.

In order to investigate the nature of the phase transition at various $p$, we use the standard Monte Carlo method and the Wang-Landau technique of simulation. Wang and Landau [11] recently proposed a MC algorithm for classical statistical models which allowed to study systems with difficultly accessed microscopic states. In particular, it permits to detect with efficiency weak first-order transitions $[12,13]$ The algorithm uses a random walk in energy space in order to obtained an accurate estimate for the density of states (DOS), call it $g(E)$, which is defined as the number of spin configurations for any given $E$. This method is based on the fact that a flat energy histogram $H(E)$ is produced if the probability for the transition to a state of energy $E$ is proportional to $g(E)^{-1}$. We

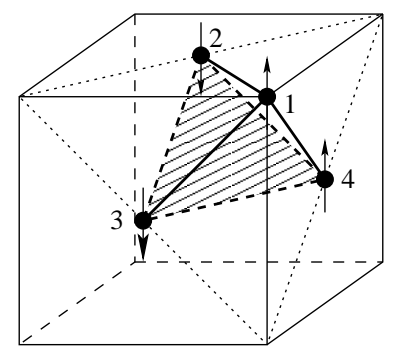

Fig. 1. The FCC cell with four sublattices. 
summarize how this algorithm is implied here. At the beginning of the simulation, the density of states $g(E)$ is unknown so all densities are set to unity, $g(E)=1$. We begin a random walk in energy space $(E)$ by choosing a site randomly and flipping its spin with a transition probability

$$
p\left(E \rightarrow E^{\prime}\right)=\min \left[g(E) / g\left(E^{\prime}\right), 1\right],
$$

where $E$ is the energy of the current state and $E^{\prime}$ is the energy of the proposed flip. Each time an energy level $E$ is visited, the DOS is modified by a modification factor $f>0$ whether the spin flipped or not, i.e. $g(E) \rightarrow g(E) f$. At the beginning of the random walk, the modification factor $f$ can be as large as $e^{1} \simeq 2.7182818$. A histogram $H(E)$ records the number of times a state of energy $E$ is visited. Each time the energy histogram satisfies a certain "flatness" criterion, the visits histogram $H(E)$ is then reset to zero, and the modification factor reduced, typically to the square root of the previous factor, to produce a finer estimate of $g(E)$. The reduction process of the modification factor $f$ is repeated several times until a final value $f_{\text {final }}$ which close enough to one. The histogram is considered as flat if

$$
H(E) \geq x \% \cdot\langle H(E)\rangle
$$

for all energies, where $x \%$ is chosen between $90 \%$ and $95 \%$ and $\langle H(E)\rangle$ is the average histogram.

The thermodynamic quantities $[11,14]$ can be evaluated by

$$
\begin{aligned}
\langle E\rangle & =\frac{1}{Z} \sum_{E} g(E) E \exp \left(-E / k_{B} T\right), \\
C_{v} & =\frac{\left\langle E^{2}\right\rangle-\langle E\rangle^{2}}{k_{B} T^{2}} \\
\left\langle M^{n}\right\rangle & =\frac{1}{Z} \sum_{E} M^{n} g(E) \exp \left(-E / k_{B} T\right), \\
\chi & =\frac{\left\langle M^{2}\right\rangle-\langle M\rangle^{2}}{k_{B} T}
\end{aligned}
$$

where $Z$ is the partition function defined by

$$
Z=\sum_{E} g(E) \exp \left(-E / k_{B} T\right)
$$

The canonical distribution at any temperature can be calculated simply by

$$
P(E, T)=\frac{1}{Z} g(E) \exp \left(-E / k_{B} T\right)
$$

In this work, we consider a energy range of interest $[15,16]\left(E_{\min }, E_{\max }\right)$. We divide this energy range to $R$ subintervals, the minimum energy of each subinterval is $E_{\min }^{i}$ for $i=1,2, \ldots, R$, and maximum of the subinterval $i$ is $E_{\max }^{i}=E_{\min }^{i+1}+2 \Delta E$, where $\Delta E$ can be chosen large enough for a smooth boundary between two subintervals. The WL algorithm is used to calculate the relative DOS of each subinterval $\left(E_{\min }^{i}, E_{\max }^{i}\right)$ with the modification factor $f_{\text {final }}=\exp \left(10^{-9}\right)$ and flatness criterion $x \%=95 \%$. We reject the suggested spin flip and do not update $g(E)$ and the energy histogram $H(E)$ of the current energy level $E$ 
if the spin-flip trial would result in an energy outside the energy segment. The DOS of the whole range is obtained by joining the DOS of each subinterval $\left(E_{\min }^{i}+\Delta E, E_{\max }^{i}-\Delta E\right)$.

\section{THE SIMULATION RESULTS}

We used the system size of $N=4 \times L^{3}$ where $L=12, p$ varies from 0 up to 1 . Periodic boundary conditions are used in the three directions. The magnetization and the susceptibility is obtained by standard Monte-Carlo simulation with the equilibrating time is $t_{e}=2,000,000 \mathrm{MC}$ steps/spin and the averaging time is $t_{a}=4,000,000 \mathrm{MC}$ steps $/ \mathrm{spin}$. The energy, specific heat and energy histograms are obtained by WL technique.

For $p=0$, it is well-known that the bulk FCC antiferromagnet with Ising spins shows a very strong first-order transition [17-20]. The phase transition in the Heisenberg model also shows a first-order character [21,22]. Other similar frustrated antiferromagnets such as the HCP antiferromagnet show the same behavior [23,24]. In Fig. 2, we show the energy and the magnetization versus temperature $T$ where the discontinuous vertical lines indicate the discontinuities at the transition temperature $T_{C}$. This is the case of pure FCC antiferromagnet. Specific heat and susceptibility as a function of temperature are shown in Fig. 3.
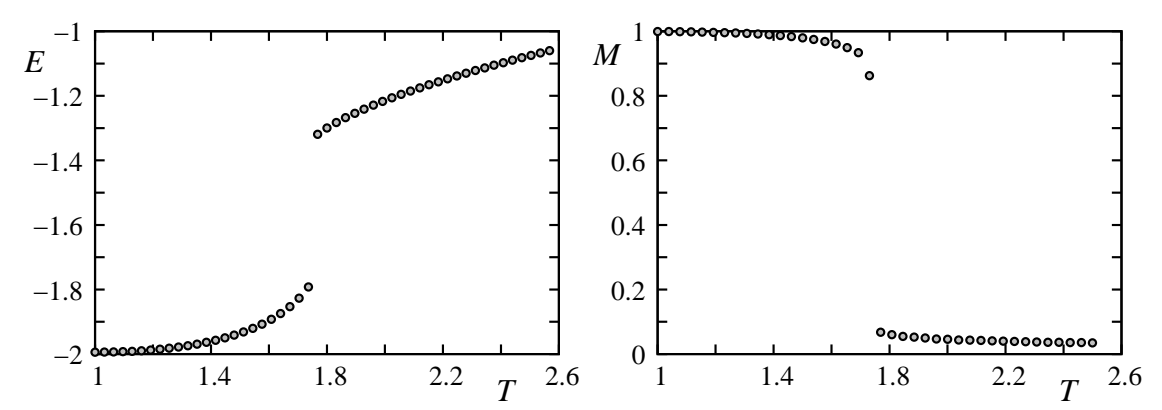

Fig. 2. Energy (left) and magnetization (right) vs temperature for $p=0$ and $L=12$.
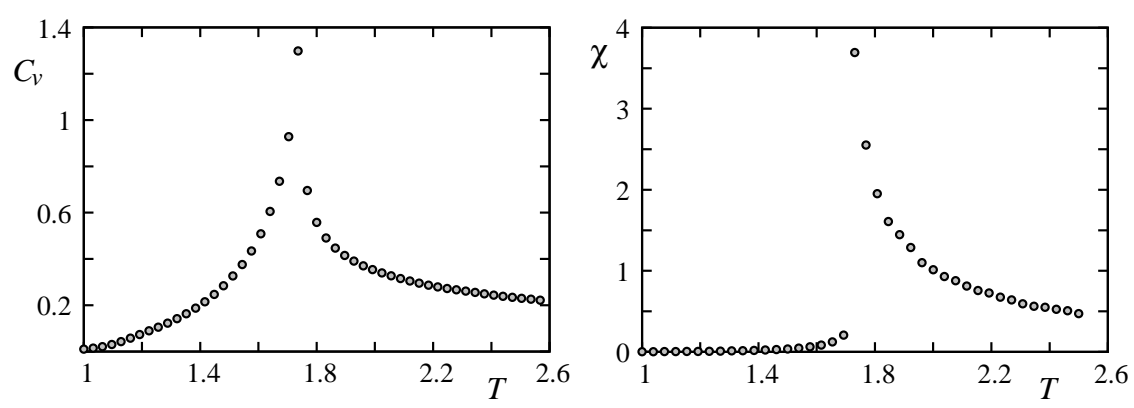

Fig. 3. Specific heat (left) and susceptibility (right) vs temperature for $p=0$ and $L=12$. 

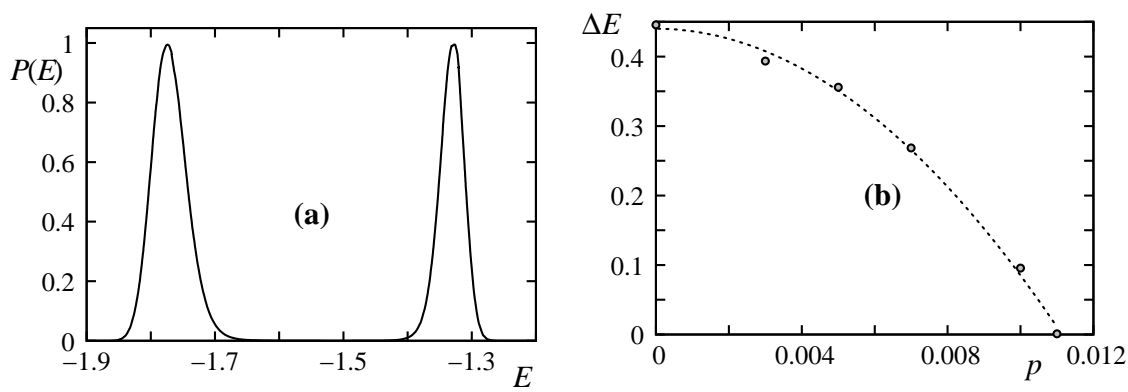

Fig. 4. (a) Energy histograms as a function of energy at $T=1.75114$ with $p=0$. (b) The latent heat vs $p$ for $L=12$.
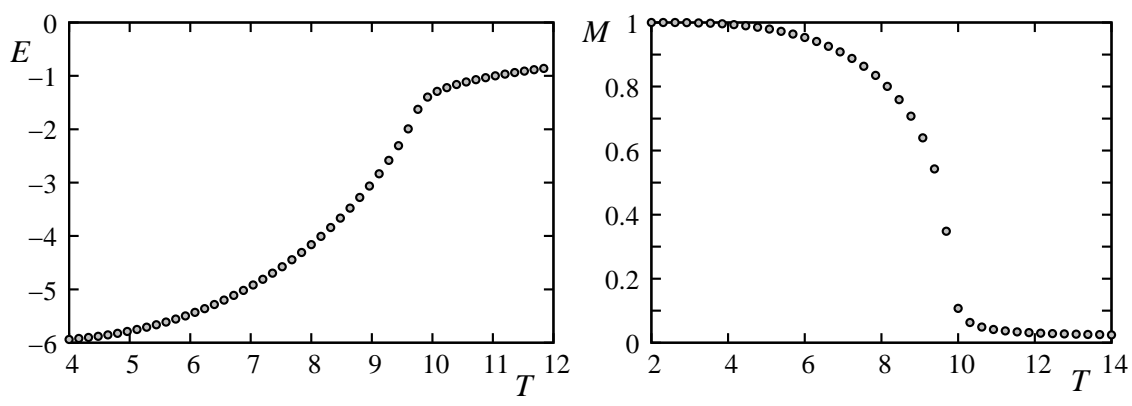

Fig. 5. Energy (left) and magnetization (right) vs temperature for $p=1.0$ and $L=12$.

Fig. 4 (a) shows a double peak of the energy distribution at $T_{C}$ which is another signature of the first-order behavior. The latent heat $\Delta E$ is defined by the energy separation of the two peaks in the energy distribution. When the transition is of second order, the energy is continuous, namely $\Delta E=0$. As shown in Fig. 4 (b) the first order transition remains as long as $p \leq 0.011$. For $p>0.011$, the phase transition is continuous.

For the case of pure ferromagnet, i.e. $p=1$, we plot in Fig. 5 the energy and the magnetization. The transition is a second-order one as expected for a 3D Ising ferromagnet.

Let us consider intermediate values of $p$ where the spin-glass phase is expected. The spin-glass phase is determined by the Edwards-Anderson freezing order parameter defined as

$$
Q=\sum_{i}^{N}\left|\sum_{t=t_{e}}^{t_{e}+t_{a}} S_{i}(t)\right| .
$$

The averaging time $t_{a}$ is taken as long as possible (at least several millions of MC per spin). The magnetization is defined as

$$
M=\sum_{t=t_{e}}^{t_{e}+t_{a}} \sum_{i}^{N} S_{i}(t) .
$$



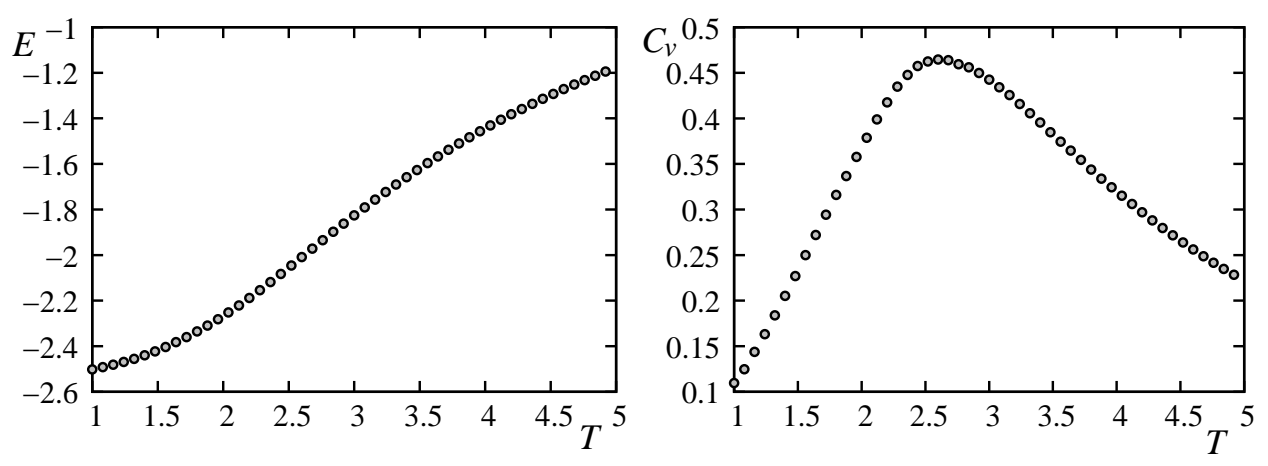

Fig. 6. Energy (left) and specific heat (right) vs temperature for $p=0.5$ and $L=12$.
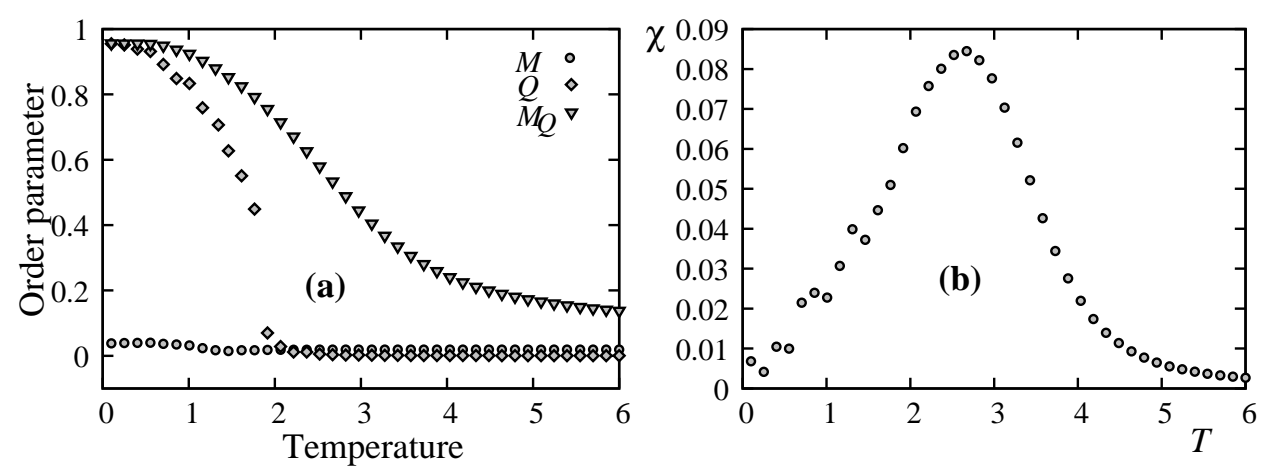

Fig. 7. (a) Magnetization $M$, order parameter $Q$, and $M_{Q}=M\left(Q_{\Delta t}\right)$ with $\Delta t=$ 30000 vs temperature for $p=0.5$. (b) $\chi=N\left(\left\langle M\left(Q_{\Delta t}\right)^{2}\right\rangle-\left\langle M\left(Q_{\Delta t}\right)\right\rangle^{2}\right) / T$ vs temperature for $p=0.5$ and $L=12$.

In a strong bond disorder, the magnetization $M$ tends to zero at any $T$ so that it cannot distinguish a frozen phase from the paramagnetic phase. We show now the result for $p=0.5$ where the bond disorder is strongest. We plot in Fig. 6 the energy and the specific heat versus temperature for $p=0.5$. quantities

To study the phase transition in the "equilibrated" regime, let us define the following

$$
\begin{aligned}
Q_{\Delta t}(t) & =\sum_{i}^{N}\left|\sum_{t}^{t+\Delta t} S_{i}(t)\right|, \\
M\left(Q_{\Delta t}\right) & =\sum_{t} Q_{\Delta t}(t) .
\end{aligned}
$$

where $\sum_{t}^{\Delta t}$ indicates a sum over $\Delta t \mathrm{MC}$ steps starting from $t$ and $\sum_{i}^{N}$ indicates a sum over the system sites. In Eq. 11, $M\left(Q_{\Delta t}\right)$ is the average of $Q_{\Delta t}$ calculated over all successive intervals of $\Delta t$ steps until the end of the total run time $t_{a} . M\left(Q_{\Delta t}\right)$ is thus averaged with $t_{a} / \Delta t=2 \times 10^{5}$ intervals (taking $\Delta t=20$ for instance). Note that $\Delta t$ is nothing but the 


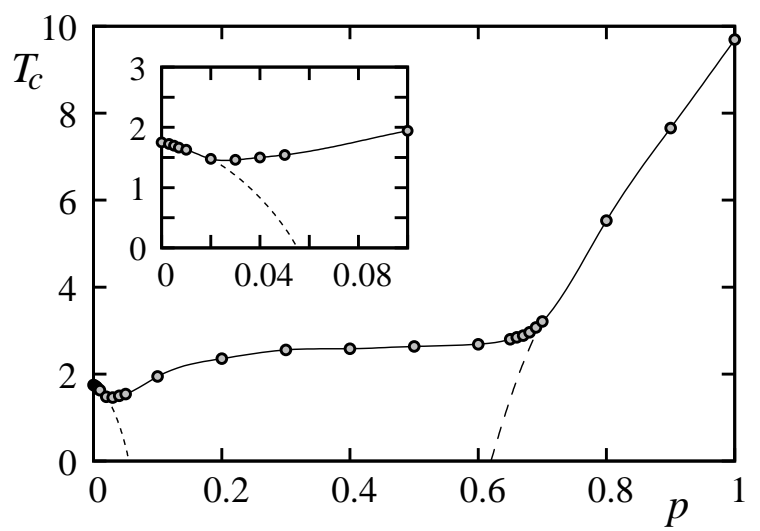

Fig. 8. The phase diagram $\left(T_{C}, p\right)$ for $L=12$.

so-called "waiting time" when measuring the magnetization. In the case of $\Delta t=1$ we obtain $M\left(Q_{\Delta t}\right) \equiv M$, and when $\Delta t=t_{a}$ we have $M\left(Q_{\Delta t}\right) \equiv Q$. As shown in Fig. 7(a), the magnetization is zero at any temperature for $p=0.5$. Therefore, it is impossible to obtain the susceptibility defined by the variance of $M$ or $Q$. However, we can do that for $M\left(Q_{\Delta t}\right): \chi=N\left(\left\langle M\left(Q_{\Delta t}\right)^{2}\right\rangle-\left\langle M\left(Q_{\Delta t}\right)\right\rangle^{2}\right) / T$. Of course, the result of $\chi$ will depend on $\Delta t$. We show an example of $\chi$ for $\Delta t=30000$ in Fig. $7(\mathrm{~b})$.

The phase diagram $\left(T_{C}, p\right)$, where $T_{C}$ is obtained at the maximum of specific heat is shown in Fig. 8. The inset enlarges the small p region. Discontinued lines are extrapolations from the antiferromagnetic-paramagnetic (AF-P) and ferromagnetic-paramagnetic (F-P) transition lines. The region below each discontinued line has a reentrant shape. This should be further clarified before a firm conclusion can be said. This is not the purpose of this work.

\section{CONCLUSIONS}

We studied in this work the phase transition behaviors of Ising spins on the FCC lattice. We found that the first-order transition has been destroyed with a tiny amount of ferromagnetic bond $p \sim 0.01$.

Using the new quantity $M\left(Q_{\Delta t}\right)$, we can obtain the susceptibility $\chi$ as a function of temperature which defined by the variance of $M\left(Q_{\Delta t}\right)$, but not by the variance of $M$ or $Q$. Therefore, the transition temperature $T_{C}$ is obtained at the maximum of $\chi$.

The phase diagram (PD) shows that the antiferromagnetic phase changes into a spin glass and then to ferromagnetic phase, then this $\mathrm{PD}$ suggests that the spin-glass phase exists in the range $0.055<p<0.62$. The $\mathrm{PD}$ is not symmetric with respect to $p$. This is due to the fact that the FCC lattice is highly frustrated at $p=0$ (antiferromagnetic) and non frustrated at $p=1$. It is not therefore surprising that the introduction of even very small amount of ferromagnetic bonds breaks immediately the long-range order. Note that the case where the pure system is non frustrated such as the simple cubic ferro- or antiferromagnets, the phase diagram $\left(T_{C}, p\right)$ is symmetric because the system is invariant with respect to the local transformation of every spin pair $\left(J_{i j} \rightarrow-J_{i j} ; S_{j} \rightarrow-S_{j}\right)$. 


\section{ACKNOWLEDGMENTS}

This work was supported by the Nafosted (Vietnam National Foundation for Science and Technology Development), Grant No. 103.02-2011.55.

\section{REFERENCES}

[1] D. Sherrington and S. Kirkpatrick, Phys. Rev. Lett. 35 (1975) 1792.

[2] S. Kirkpatrick and D. Sherrington, Phys. Rev. B 17 (1978) 4384.

[3] A. T. Ogielski, Phys. Rev. B 32 (1985) 7384.

[4] R.M.C. de Almeida, N. Lemke, I. A. Campbell, J. Magnetism and Magnetic Materials 226-230, $1296(2001)$

[5] D. C. Johnston, Phys. Rev. B 74 (2006) 184430.

[6] Alain Billoire and I. A. Campbell, Phys. Rev. B 84 (2011) 054442.

[7] L. Lundgren, P. Svedlindh, P. Nordblad, and O. Beckman, Phys. Rev. Lett. 51 (1983) 911; L. Lundgren, P. Nordblad, and P. Svedlindh, Phys. Rev. B 34 (1986) 8164; L. Lundgren, in Relaxation in Complex Systems and Related Topics, edited by I. A. Campbell and C. Giovannella, Plenum Press, New York, 1990.

[8] E. Vincent, Lecture Notes in Physics, Springer-Verlag, Berlin, 2007, Vol. 716, pp. 7 - 60.

[9] Itsuko S. Suzuki and Masatsugu Suzuki, Phys. Rev. B 78, 214404 (2008).

[10] R. M. Pickup, R. Cywinski, C. Pappas, B. Farago, and P. Fouquet, Phys. Rev. Lett. 102 (2009) 097202.

[11] F. Wang and D. P. Landau, Phys. Rev. Lett. 86 (2001) 2050; Phys. Rev. E 64 (2001) 056101.

[12] V. Thanh Ngo and H. T. Diep, J. Appl. Phys. 103 (2008) 07C712.

[13] V. Thanh Ngo and H. T. Diep, Phys. Rev. E 77 (2008) 031119.

[14] G. Brown and T. C. Schulhess, J. Appl. Phys. 97 (2005) 10E303.

[15] B. J. Schulz, K. Binder, M. Müller, and D. P. Landau, Phys. Rev. E 67 (2003) 067102.

[16] A. Malakis, S. S. Martinos, I. A. Hadjiagapiou, N. G. Fytas, and P. Kalozoumis, Phys. Rev. E 72 (2005) 066120.

[17] M. Phani, J. L. Lebowitz and M. H. Kalos, Phys. Rev. B 21 (1980) 4027.

[18] T. L. Polgreen, Phys. Rev. B 29 (1984) 1468.

[19] D. F. Styer, Phys. Rev. B 32 (1985) 393.

[20] H. T. Diep, A. Ghazali, B. Berge and P. Lallemand, Euro-physics Letters 2 (8), 603 (1986).

[21] H. T. Diep and H. Kawamura, Phys. Rev. B 40 (1989) 7019.

[22] M. V. Gvozdikova and M. E. Zhitomirsky, JETP Lett. 81 (2005) 236.

[23] H. T. Diep, Phys. Rev. B 45 (1992) 2863, and references therein.

[24] Danh-Tai Hoang and H. T. Diep, Phys. Rev. E 85 (2012) 041107. 